\title{
The Analysis of the Difference of Land-use Landscape Patterns between the Jinsha River Dry-hot Valley and the Redbed of Middle Yunnan Province-Based on the Typical Basins of the Redbed of Middle Yunan Province
}

\author{
Xian Dai ${ }^{1, a}$, Linpei Huang ${ }^{1,2, b *}$ and Yunchuan Dai ${ }^{1, c}$ \\ ${ }^{1}$ School of Tourism and Geographical Science, Yunnan Normal University, Kunming 650500, China. \\ ${ }^{2}$ Key Lab of Plateau Lake Ecology and Global Change, School of Tourism and Geography, Yunnan \\ Normal University, Kunming 650500, China

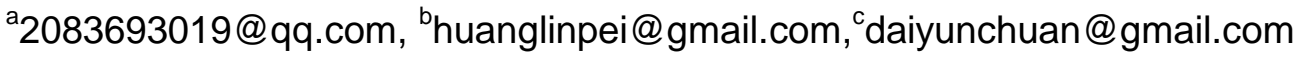 \\ *The corresponding author
}

Keywords: Jinsha river dry-hot valley; Redbed of the middle Yunnan province; Land-use; Landscape patterns; Fragstats

\begin{abstract}
The landscape patterns of the current land-use on the redbed of middle Yunnan province Was studied in this paper. Based on the landscape pattern index and the spatial analysis technics ArcGIS 10.0, the landscape patterns of the land-use on the redbed of middle Yunnan province was analyzed, and the difference of the landscape patterns of the land-use between the Jinsha River dry-hot valley and the redbed of middle Yunan Province was discussed.The results indicated that: (1) Forest,the dominant landscape, occupied $53.6031 \%$ of the redbed of middle Yunnan province,followed by cultivated land; (2) The obvious spatial differential of landscape patterns between the Jinsha River dry-hot valley and the redbed of middle Yunnan province did exist. The basins in the Jinsha river dry-hot valley showed the low SHDI, SHEI, and high CONT, DI; (3) The basins on the redbed of middle Yunnan province showed the high SHDI, SHEI, and low CONT, DI; (4) The fragmentation index of the basins on the redbed of middle Yunnan province were higher, indicating that human activities showed a significant positive correlation with the fragmentation of landscape.
\end{abstract}

\section{Introduction}

Since the 1990s, driven by IGBP and IHDP, the land-use or the change of land-cover has become an important field in the study of global environmental change. Currently, the study of land-use has involved in such fields as analysis of landscape pattern of land-use, spatial-temporal evolution, ecological environment effect and driving mechanism ${ }^{[1]}$. The analysis of the difference of landscape patterns of land-use has the scientific significance on strengthening the protection of land resources, combating unfair exploitation, and improving the ecological environment. Landscape patterns refer to the arrange form in space of sizes and shapes of landscape elements. It is not only a concrete form of landscape heterogeneity, but also the result of various ecological processes work at different scales ${ }^{[2]}$. The analysis of landscape patterns of land-use is helpful for the deeper understanding of the characteristics of landscape patterns of land-use. Scholars at broad have done lots of research on the relationship between landscape patterns of land-use and landscape processes ${ }^{[3-5]}$. On the contrary, domestic scholars mainly explore the characteristics of landscape patterns of land-use, spatial-temporal evolution, driving mechanism, and discuss the patterns and evolution of large scales of Chinese land-use. Meanwhile, some scholars also discuss the current landscape patterns of land-use of medium and small scale areas (cities/districts/counties, watersheds) ${ }^{[6]}$. At present, there is no scholar studying the difference of landscape patterns of land-use in dry-hot valley and the plateau. 
The redbed of middle Yunnan is located in the central part of Yunnan province, bounded by Jin Shanjiang-Ailao mountain fault zone in the east, Tang Lang-West fault zone in the west, $27^{\circ} 92^{\prime} \mathrm{N}$ to the north, and $23^{\circ} 28^{\prime} \mathrm{N}$ to the south. It includes Chuxiong, the east of Lijiang, Binchuan, Xiangyun, Midu, Xinping, Yuanjiang, with an area of about 53325.89 square kilometers. The area, the dome-shaped plateau consisted of purple sandstones, lies in high north and low south, 1300-1950 meters in altitudes. The distribute area of forest is rather wide, and belongs to one of the major forest areas in Yunnan province, represented with the plateau semi-humid evergreen broad-leaved forest and Yunnan pie forest. The paper, selecting 2014 global 30 meters land cover data of middle Yunnan redbed as the study data, basing on the platform GIS, using the analysis software of landscape pattern -Fragstats3.4, quantitatively describes the general characteristics of current landscape patterns of land-use in middle Yunnan redbed. It analyzes the spatial distribute rule of landscape patterns of land-use in terms of the basin of Jinsha river dry-hot Valley and redbed from four aspects: diversity, heterogeneity, aggregation and fragmentation, in order to provide a theoretic basis for the scientific land-use in Yunnan province and the macro-policy of government.

\section{Data Resource and Research Method}

Data Resource The remote sensing image with 30 meter resolution is considered the best measure to describe changes of global land cover and its scale. The study data of global 30-meter land-cover is downloaded from the website:

http://www.globallandcover.com/GLC30Download/index.aspx. GlobalLand30 is the fruit of Chinese 863 program- remote image of global earth's surface and essential technique research. The data is derived from the image data of American TM5、ETM+ and Chinese HJ 1, and is formed on the basis of such a comprehensive method as the classification of pixel- the extraction of object- the check of knowledge, including nine first-class types of land-cover, namely cultivated land, forest, grassland, shrub, wetlands, water body, artificial surface, bare land, ice and snow.

Research Method The study uses the landscape patterns index to demonstrate the spatial characteristic of current landscape patterns of land-use of middle Yunnan redbed, quantitatively reflecting the allocation characteristics of landscape pattern and space of land-use. Referring to many landscape pattern indexes [7-8], that scholars have put forward, combining specific characteristic of research areas with the practical need of research, and selecting the significant indexes, it chooses five landscape patterns indexes to describe the characteristic of landscape pattern of land-use of middle Yunnan redbed from the aspects of diversity, heterogeneity, aggregation and fragmentation. The indexes and its ecological significance are seen as table ${ }^{[1]}$. Firstly, the surface chart of research areas is changed into the form of Arc Grid and size of $5 \mathrm{~m} \times 5 \mathrm{~m}$ by means of ArcGIS10.0, in order to meet the accuracy of research. Then, these data are imported in the software Fragstats 3.4 and count the value of each landscape.

Table 1 The general landscape indexes

\begin{tabular}{|c|c|c|c|}
\hline Landscape & Englis & The formula & Ecological significance \\
\hline
\end{tabular}

Shannon diversity SHDI $\quad S H D I=-\sum_{i=1}^{m}\left(p_{i} \times \ln p_{i}\right)$ index
In a landscape system, land use, the more abundant, the higher the degree of fragmentation, the greater the information content of its uncertainty, Calculate the SHDI value is higher, $\mathrm{SHDI}>0$. 
Table1 before

Shannon evenness index

SHEI $\quad$ SHEI $=\frac{-\sum_{i=1}^{m}\left[P_{i} \times \ln \left(P_{i}\right)\right]}{\ln m}$

Dominance index

DI

$$
D I=H_{\max }+\sum_{k=1}^{n} P_{k} \log _{2} P_{k}
$$

$$
D I=H_{\max }+\sum_{k=1}^{n} P_{k} \log _{2} P_{k}
$$

Fragmentati on index

FI

$$
F I=\left(N_{P}-1\right) / N_{C}
$$

Agglomerat ion index
$\mathrm{CONT} \quad \mathrm{CONT}=\left(\sum_{i=1}^{n} \sum_{j=1}^{n} \frac{P_{i j} \log _{2} P_{k}}{2 \log _{2} n}\right) \times 100$
Describe the landscape of each component in the distribution uniformity, $0 \leqslant \mathrm{SHEI} \leqslant$ 1 。 SHEI=0 indicates that landscape consists only of a plaque, no diversity; SHEI $=1$, indicates that uniform distribution for various types of landscape patches, diversity is the largest

Refers to the landscape type structure of a certain type or some landscape types in the degree of the dominant. If the dominance index DI, the greater the landscape dominance index is higher

The fragmentation degree of landscape is divided, $0 \leq \mathrm{FI} \leq 1$. FI $=0$, Said there was no destruction of landscape, $\mathrm{FI}=1$ indicates that the landscape is completely destroyed

Index reflect the size of the landscape in the aggregation degree of different plaques and extending trend, also can reflect the landscape space configuration characteristics of the component

Table 2 The general characteristics of the landscape pattern on the redbed of middle Yunan Province

\begin{tabular}{cccccc}
\hline Type & CA/ha & PLAND/\% & NP/singleton & MPS/ha & PD/100ha \\
\hline Forest & 2858432.6700 & 53.6031 & 220534 & 12.9614 & 4.1356 \\
Shrub & 494974.1700 & 9.2821 & 456191 & 1.0850 & 8.5548 \\
Grass & 730078.9200 & 13.6909 & 354002 & 2.0624 & 6.6385 \\
Barren & 17976.3300 & 0.3371 & 17493 & 1.0726 & 0.3280 \\
Cultivated land & 1163667.8700 & 21.8218 & 20119 & 57.8392 & 0.3773 \\
Water body & 28390.5000 & 0.5324 & 3894 & 7.2908 & 0.0730 \\
Artificial & 38194.7400 & 0.7163 & 2028 & 18.8337 & 0.0380 \\
surface & 873.7200 & 0.0164 & 89 & 9.8171 & 0.0017 \\
Wet land & & & & & \\
\hline
\end{tabular}

\section{The Analysis of Landscape Patterns of Land-use}

The General Characteristics of Landscape Patterns. Based on the GIS, the study makes a relevant statistical processing and analysis different types of landscape in terms of the data of 2014 land-use in middle Yunnan redbed, and uses Fragstat3.4 to count relevant landscape pattern indexes. It can be clearly seen that forest account for $53.6031 \%$ of the whole landscape area in middle Yunnan redbed, 
and is much higher than other types of landscape, reflecting that the forest is the main types of land-use in the region. The development of economy in the region is lag behind eastern area lag. National poverty area and much agricultural population leads that the area of cultivated land is large and artificial surface (construction land, traffic land) is small, and that cultivated land account for $21.8218 \%$ among the whole area. Due to the large area of red layer district in middle Yunnan distribution, red-layer is mostly weak and contains water rock, and groundwater under the area is extremely poor. Therefore, the ratio of water and wetland in the region is low.

There is a remarkable difference in the landscape patterns of land-use of basin on Jinsha River dry-hot and the redbed. Different types of landscapes in the region take on various rules in the numbers and space distribution. From the figure 1 and figure 2, it can be seen that the basic rules of landscape patterns of basins are as follows: 1)Cultivated land is the most important landscape type in all basins, the main type of landscape of land-use, and the natural environmental basis that maintains the stability of whole ecological system in the region. It is widely distributed in all basins, among which the Jinsha River dry-hot is the main area. 2) Forests and bushes mainly distribute in the upper redbed, where the forest of Qiaodian dam and bush of Luochuan dam account for the largest areas, with values up to $26.5056 \%$ and $20.7151 \%$ respectively. 3) The artificial surface and grass within each basin also relatively have a wide distribution,whose difference is not significant. 4) Water mainly lies in the region of Jinsha River dry-hot Valley. Some river and lake are at odds located at the upper redbed, but take up a relatively small proportion.5)Wetlands and bare land distribute in several basins, which cannot correctly reflect the landscape differences of types of land-use of Jinsha River dry-hot Valley and upper redbed area.

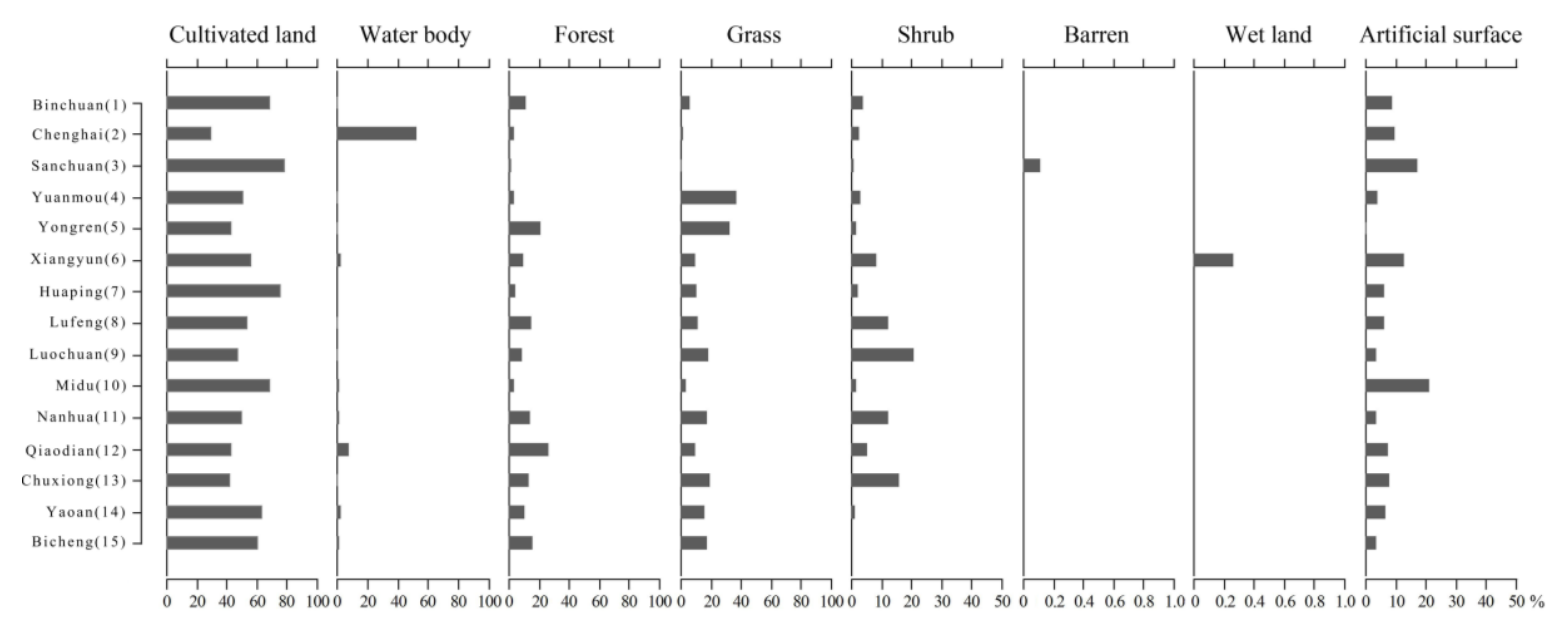

Figure 1. The percentage of the landscape types of the typical basins

The Rules of Spatial Distribution of Landscape Due to the differences of natural geographical environment (terrain, weather and river system) and socioeconomic conditions (industry, population and culture), the spatial distribution of types of landscape in the region take on great differences, especially in the landscape's diversity, fragmentation and heterogeneity. The paper, on the basis of platform Fragstat3.4, attempts to explore and study the spatial distribution of land-use landscape of middle Yunnan redbed from these basic views of diversity, heterogeneity, aggregation and fragmentation.

The Distribution of Landscape Diversity Index of landscape diversity is mainly reflects the complexity and variability of all landscapes in the landscape pattern of land-use. In terms of the landscape diversity, the average value of basins situated in the dry-hot valley of middle Yunnan redbed is 1.0610, and those in the plateau is 1.2813. In all basins, the landscape diversity index of Chuxiong on the plateau is the biggest, which is up to 1.4955.The index of Sanchuan on the dry-hot valley is the smallest, up to the 0.6669.Index of other basins is between 0.8641 and 1.4904. Through 
the analysis of landscape diversity index, it can be drawn the following basic rules that the landscape diversity index has in general the tendency of increasing from dry-hot valley to basin on the plateau. The index of basin near to the Jinsha River dry-hot valley is clearly lower than the one of the plateau. The main reason is that the terrain of Jinsha River dry-hot valley is flat and the soil is fertile, contributing to the cultivation and plant of farming. Meanwhile, the population of the region is relatively dense, and the construction speed of cities and towns is fast, and the scale of construction land is large. The main types of landscape in the terrain are cultivated land and construction land. On the contrary, because of the high terrain of basins on the plateau, shortage of water and poor soil, the production condition of agriculture is badly-off. Also, the density of population is low. The impact of human activity on the landscape of land-use is small, and landscape diversity index in general is high. It fully convinces that the impact of human activity on the landscape diversity of land-use is considerable and that the inverse relationship is significant.

The Distribution of Landscape Heterogeneity Landscape heterogeneity refers to the inhomogeneity and complexity in time and space of land-use landscape, which mainly symbolized by means of evenness index and dominance index. The two indexes are used to describe the control degree of several landscapes among the land-use landscapes, and can be tested by each other. The highest dominance index is Sanchuan dam, up to 0.6573, and the lowest one is Chuxiong, up to 0.1654 and index of other basins is about between 0.1682 and 0.5178 . Through the analysis of landscape dominance index, it can be drawn the following basic rules that the landscape dominance index has in general the tendency of decreasing from dry-hot valley to basin on the plateau. Jinsha River dry-hot valley is close to the water resource and is suitable for the planting of agriculture. Cultivated land is the main landscape in the valley. Therefore, cultivated land has a prior position, while the basin on the plateau is inferior, because of the poor soil, shortage of water, poor condition of farming. Compared with the Jinsha River dry-hot valley, the cultivated land on the plateau is small, and forest and bush are big, and prior landscape is weak, and dominance index of land-use landscape is accordingly low.

The average value of evenness index of land-use landscape of basins on the middle Yunnan redbed dry-hot valley is 0.5793 , and the average index of basins on the plateau is 0.5793 . The highest index is the Chuxiong dam, up to 0.8346, while the lowest index is the Sanchuan dam, up to 0.3427.The index of other dams is between 0.4822 and 0.8318 .Through the analysis of landscape evenness index, it can be drawn the following basic rules that the index of land-use landscape on the Jinsha River dry-hot is significantly lower than one on the basin. The main reason is that the farming condition of dry-hot valley is prior to the condition of basin. Cultivated land is dominant in the landscapes. Conversely, all types of landscapes on the basin take on a balanced tendency in space distribution, and landscape evenness index is accordingly high.

The Distribution of Landscape Aggregation Landscape aggregation index refers to the degree of aggregation and characteristics of land-use landscape in time and space. Average value of land-use aggregation index of basin on the middle Yunnan redbed dry-hot valley is 61.1165, while the average value of one on the plateau is 49.9781. The highest aggregation index is Sanchuan dam on the Jinsha River dry-hot valley, up to 77.8890, and the lowest one is Chuxiong dam on the plateau, up to 0.1654 and index of other basins is about between 43.3436 and 65.4985 .

Through the analysis of landscape aggregation index, it can be drawn the following basic rules that the aggregation index of land-use landscape on the Jinsha River dry-hot valley is higher than that on the basin. In the region of Jinsha River dry-hot valley, land-use landscape consists of several dominant landscapes. Due to the factors such as terrain and water source, the area of cultivated land is largely distributed. Large scales of space distribution of cultivated land make the characteristics of land-use simple, the odd area of landscape large, the degree of aggregation index high and the land-use structure simple.Conversely, the degree of priority of land-use on redbed is relatively low, and cultivated land and forest take on the balanced tendency in space, leading to the lower aggregation of land-use. 

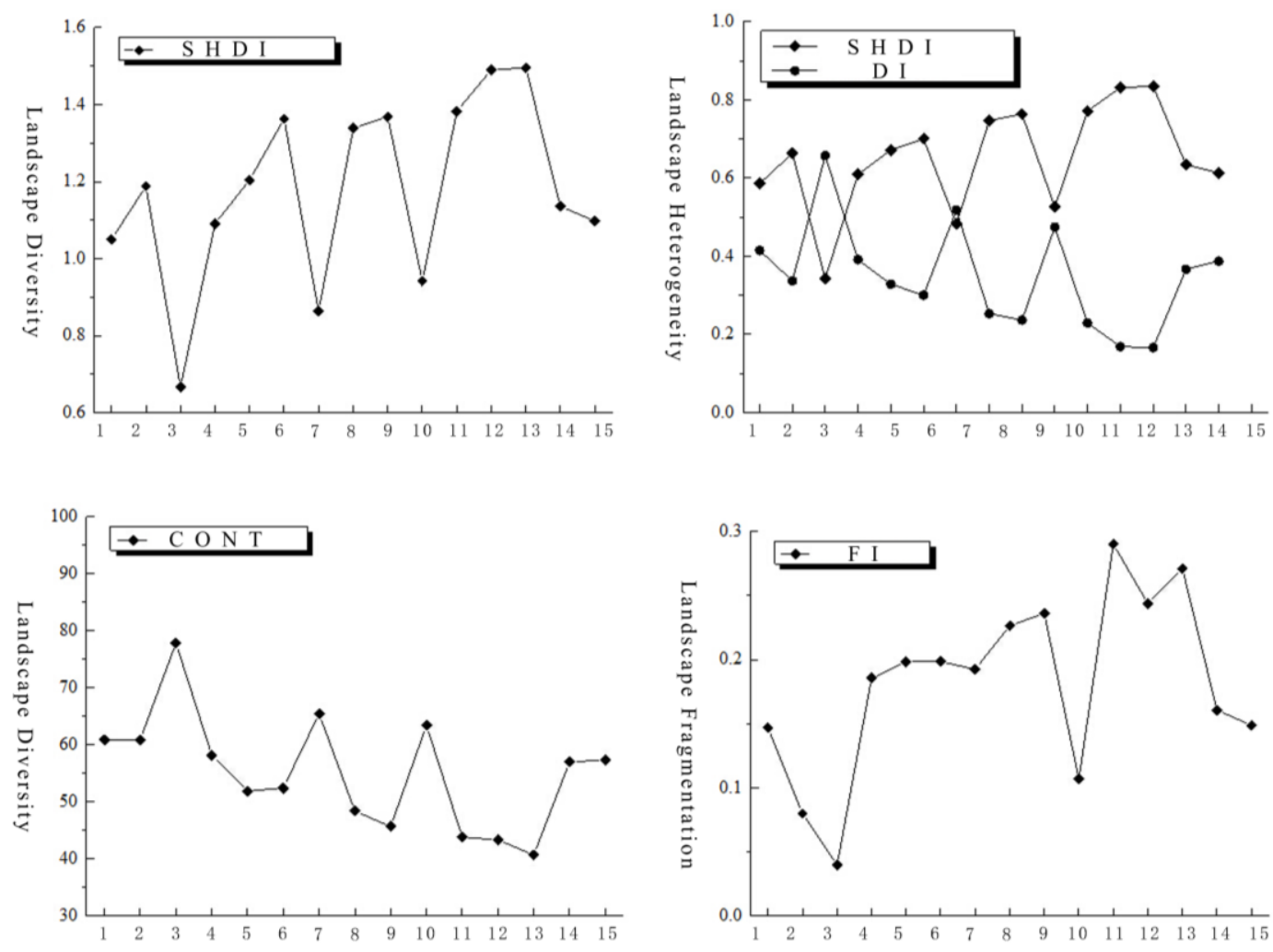

Figure 2. The line graphs of the landscape pattern on the redbed of middle Yunan Province

The Distribution of Landscape Fragmentation Landscape fragmentation refers to the fragmentation of land-use and the complexity of landscape structure, which can reflect the heterogeneity of landscape. The higher of landscape fragmentation is, the higher of landscape heterogeneity is. Average value of land-use fragmentation index of basin on the middle Yunnan redbed dry-hot valley is 0.1488 , while the average value of one on the plateau is 0.2101 . The highest fragmentation index is Nanhua dam on the plateau redbed, up to 0.2903 , and the lowest one is Sanchuan dam on the Jinsha River dry-hot valley, up to 0.0399 and index of other basins is about between 0.0799 and 0.2712 . Through the analysis of landscape fragmentation index, it can be drawn the following basic rules that the fragmentation index of plateau redbed is higher than that of Jinsha River dry-hot valley. Because of the concentrated distribution of all types of landscape elements on the Jinsha River dry-hot valley, the scattered distribution of those on the plateau, the fast speed of socioeconomic development of redbed, and larger percentage of artificial surface (construction land, traffic land), the fragmentation of land-use landscape is remarkable. This fully demonstrates that increasing of human's development activities can also lead to increase the degree of land-use landscape fragmentation. The relationship between them is significantly positive.

\section{Conclusions}

The paper, taking the coverage data of 2014 global 30 meters surface of Yunnan redbed as the research object, based on spatial analysis platform GIS, analyzes the general spatial characteristics of land-use landscape of Yunnan redbed from eight landscapes such as cultivated land,water, forest and artificial surface, and then selects relevant landscape index, based on the use of Fragstat3.4,and explores the spatial distribution of land-use landscape in terms of the basins on the Jinsha River dry-hot valley and the redbed from four aspects of diversity, heterogeneity, aggregation and fragmentation. The basic conclusions are drawn as follows: 1) the dominant landscape of middle Yunnan redbed is forest which covers $53.6031 \%$ of whole area, followed by cultivated land. Forest is mainly distributed in the hilly land, while cultivated land in the edge of river and lake where terrain is flat, soil is fertile and water source is abundant, due to the restriction of terrain and water source.2)In 
terms of landscape patterns of land-use of basins on the Jinsha River dry-hot valley and redbed, the characteristics of spatial distribution are obvious. Landscape diversity and evenness of basins on the Jinsha River dry-hot valley is lower, while the index of aggregation and dominance is higher. On the contrary, land-use landscape diversity and evenness of basins on redbed is higher, while the index of aggregation and dominance is lower. Higher fragmentation of basins on redbed manifests the positive relationship between human activities and landscape fragmentation of land-use. Human activity is an essential reason leading to the increasing of landscape fragmentation. In the protection of land of middle Yunnan redbed, and at the same time, the reasonability of exploitation and utilization of land should be reinforced, in order to realize scientific utilization and sustainable development of land in the region.

\section{Acknowledgements}

This work is supported by the Nature Science Foundation of China(41302151).

\section{References}

[1] ZHANG Rong-tian, ZHANG Xiao-lin, LI Chuan-wu.Analysis on the Landscape Spatial Patterns of Land-use in Zhenjiang,J.The College of Geography Science, Nanjing Normal University, China, 2012, 32(9):132-137.

[2] CHEN Wen-Bo,XIAO Du-Ning,LI Xiu-Zhen,Institute of Applied Ecology,Chinese Academy of Sciences. Shenyang, 110016, China. Acta Ecoloqica Sinica. 2002, 22(7):1135-1142

[3] Turner MG, Spatial and temporal analysis of landscape patterns, J. Landscape Ecology. 1990, 4(5):21-30.

[4] Fujihara M, Kikuchi T, Changes in the landscape structure of the Nagara River Basin, central Japan, J. Landscape and Urban. Planning, 2005, 7(4):271-281.

[5] Mander, Rob.H.G. Jongmsn. Huma impact on rural landscapes in central and northern Europe, J. Landscape and Urban Planning, 1998, 41(5):149-153.

[6] CHEN Li-hui, CHEN Zhi-biao, CHEN Zhi-qiang, ZOU Ai-ping.Quantitative Analysis on Landscape of Land Use in the Small Watershed of Zhuxi Riwer, J. College of Geographical Science, Fujian Nermal. University, China, 2009, 16(1):66-70.

[7] XU Yuantao. Identifying Landscape Pattern Metrics for the Hani Terrace in Yunnan, China, J. Journal of Resources and Ecology, 2013, 4(3):212-219.

[8] CHEN Wenbo, XIAO Duning and LI Xuzhen. Classification, application, and creation of Landscape indices, J. Institute of Applied Ecology, Chinese Academy of Sciences. Shenyang, 110016, -Chin. J. Appl. Ecil, 2002, 13(1):121-125. 